Review

\title{
DNA Vaccination Associated with Eliminating Inheritance Risks during Embryo Development: A Review
}

\author{
Ria Margiana \\ Department of Anatomy, Faculty of Medicine, Universitas Indonesia, Jakarta, Indonesia
}

\author{
Article history \\ Received: 15-04-2016 \\ Revised: 23-05-2016 \\ Accepted: 25-05-2016 \\ Email: riamargiana@yahoo.com
}

\begin{abstract}
The process of immunization using modified DNA is essential whenever scientists desire to trigger an artificial response in the host's body to improve immunity. The use of DNA vaccines awakens the somatic cells of the host to produce antigens that will assist the body to prepare to fight infections through preventive measures triggered by the vaccines. These biological inventions have assisted in controlling diseases as well as eliminating some health conditions. Scientific analyses have shown how the plasmid DNA could trigger an artificial immune response in living organisms when associated with the antigens that are plasmidencoded. Such revelations have demonstrated that this invention has a possible therapeutic application as vaccination to prevent numerous pathogens and their related infections. This paper reviews the concept of DNA vaccination and its effects on embryogenesis. The paper first discusses an overview of DNA vaccines and relates it to the production and expression processes. The effects on embryo development have also been described. This review also explains the advantages and disadvantages related to DNA vaccines' administration and the mechanisms employed to enhance plasmid effectiveness.
\end{abstract}

Keywords: DNA Vaccination, Embryogenesis, Transfection, Antibodies, Major Histocompatibility Complex (MHC) and Antigen Presenting Cells (APC)

\section{Introduction}

One of the most recent discoveries in the scientific developments for humans and animals is the concept of Deoxyribonucleic Acid (DNA) vaccination. The process of immunization using a modified DNA is essential whenever scientists desire to trigger an artificial response in the host's body to improve immunity. The use of DNA vaccines awakens the somatic cells of the host to produce the antigens that assist the body to prepare to fight infections through preventive measures triggered by the vaccines. Through DNA vaccination, dominant infections of animals have been controlled and the hope to eradicate human infections and diseases using the same technique is high. In humans, the aspect of DNA vaccination is not entirely advanced and experimental studies are being conducted to ascertain the possibility of existing solutions to eliminate common bacterial, viral and parasitic infections in humans as it has been done with animals. The use of the modified DNA vaccines is associated with a possible control and treatment for tumours and other complex somatic and physiological complications (Ming-Derg et al., 2009). More investigations that are experimental are expected in this area to reveal more scientific mechanisms for managing health complications in animals and humans.

Among humans, the need to enhance the existing vaccines to better versions has enabled scientists to design different experimental investigations. Over several years, one of the aims in this field of DNA vaccination has been to improve the species of vaccines used. The vaccines that have been created have more reliable potential compared to the previous versions. These biological inventions have assisted in controlling diseases as well as eliminating some health conditions. The conventional vaccines are associated with lack of medical sustainability. However, DNA vaccines have the potential to control multiple and spontaneous immune responses in the body. It is essential to note that DNA vaccines have a significant effect on the development of the embryo and the pregnancy period whenever they are administered to expectant animals. 
The development of the embryo depends on the processes of the host and therefore DNA alterations may affect growth and development. The administration of DNA vaccines to pregnant animals changes the genetic makeup of the blastocyst as it develops into a complete organism (Barouch et al., 2000). It is also essential to recognise that the process of producing DNA vaccines is a complex and sensitive procedure that requires more understanding and analysis. In this paper, we review the gene delivery methods, the DNA production processes and techniques and the effects of DNA vaccines on embryogenesis.

Increasing scientific knowledge and the understanding of DNA modification have enabled scholars to invent preventive measures for human and nonhuman infections. One such area includes DNA vaccination in animals. The process involves the injection of animals with a modified DNA to prevent infections by triggering the production of antigens by the somatic cells, which improves their immunity through an artificial protective response. The frequency of the scientific studies conducted in this field shows how there is a constant desire for full exploitation of necessary techniques that will assist in solving the scientific dilemmas through DNA vaccination, both in humans and animals. Although the complexity associated with the inventions regarding humans are relatively few, there are efforts toward reasonable findings. It is essential to recognise that DNA vaccination in special conditions such as pregnancy and embryogenesis is crucial and delicate; therefore, there is the need for more knowledge, experimental analysis and investigation of literature regarding these particular cases. The use of DNA vaccination has effects on pregnancy and embryogenesis because of the subtle and sensitive nature of the process as well as the nature of DNA production processes.

\section{Overview of Gene Vaccination}

Scientific analyses have shown how the plasmid DNA could trigger an artificial immune response in living organisms when associated with the antigens that are plasmid-encoded (Ulmer et al., 1993). Such revelations have demonstrated that these inventions have a possible therapeutic application as human vaccination to prevent numerous pathogens and their related infections (Ingolotti et al., 2010). Besides, it is possible for such techniques to be used to generate autoimmunity and allergy management and control (Silva et al., 2009; Spiegelberg et al., 2002). The vaccines have been advanced to be applied in the management of other complicated medical conditions in genetics such as neurological disorders and cancer (Shimamura et al., 2011; Alam and McNeel, 2010). The technology of gene modification has also been employed in the veterinary field to help the control of the West Nile virus, hematopoietic necrosis virus and melanoma in horses, salmon and dogs, respectively (Redding and Weiner, 2009). Among the applications in humans, the licensing of DNA vaccines is limited because of the possible implications that have not been thoroughly investigated and approved.

It is important to note that using DNA vaccines is less costly and reliable compared to using traditionally developed proteins that are utilised to fight pathogenic viruses without developing a sustainable protective mechanism for somatic tissues. The antigens can also be easily modified and an inclination to the structural changes of the targeted pathogens is carried out. However, the concerns raised against the immunogenicity of DNA applications are that they tend to be slower and weaker compared to the traditional immunity proteins. Therefore, the process of the immunogenicity viability has been improved through the changes within the promoters. The use of the sequences of the codon antigen and the integration of the genetic adjuvants like cytokines and activation molecules have also assisted in increasing the efficiency of the immunogenicity. Besides, other approaches employed to boost the action of DNA vaccines have included the variation of the strategies for priming and boosting the vaccination through the modification of the routes of administration (Saade and Petrovsky, 2012).

Moreover, the evaluations of DNA molecular structures and mechanisms have enabled the establishment of a variety of vaccines to be used in veterinary applications and humans. The TANK-binding kinase (TBK) and the stimulator of the interferon gene (STING) were the first to be identified as the molecular components of the DNA strands, which are essential for the induction of sustainable immune responses by the generated vaccinations. The double-strand DNA (dsDNA) has been found to be a crucial ligand of the signaling cascade of the STING-TBK1 (Desmet and Ishii, 2012). Such revelations have showed how the immune responses induced by the dsDNA signaling result in an inductive process for the antigen encoded by the DNA like the adjuvant. However, the concept of DNA sensing and segregation is still complicated and not yet fully exploited.

\section{Mode of Action and Methods of DNA Vaccination}

\section{Mode of Action}

DNA vaccines have the potential to trigger both the humoral and cellular responses meant to boost immunity of the body of the organism. The responses 
are against the encoded plasmid antigens that depend on the performance of the respective DNA vaccines. The introduction of plasmid DNA into somatic tissues such as the skin, muscles, or nasal cavity allows the plasmids to enter the body cells. When plasmid DNAs get into the somatic cells, they translocate to the interior and reside within the nucleus where the organism's response structures express the relevant antigen. Several of the myocytes and the antigen presenting cells (APCs), like the macrophages and dendritic cells, acquire and reflect the plasmid DNAs. In this scenario, the antigen proteins have been noted to be degraded and presented by the major histocompatibility complex I (MHC-I) found on a subgroup of cells responsible for the immunity of the body (Faurez et al., 2010). The cells can also produce the expressed antigens through the secretion of the proteins that could be caused by the apoptosis of the cells that have been transfected. Finally, the antigen proteins within the body tissues are displayed by the internal APCs on the molecules of MHC-I and MHC-II (Rekvig and Nossert, 2003). This formation then triggers the naïve $\mathrm{B}$ cells within the lymph node system.

\section{Methods of DNA Vaccination}

The choice of the methods employed by the genetic experts to administer DNA vaccines has been noted to be associated with limited shortcomings and possibility of compromise. The techniques used are supposed to depict low transfection efficacy. Besides, the methods should guarantee the limited levels of APC recruitment within the area of administration. The most common method used in DNA vaccination is the intramuscular electroporation (imEPT). The method is efficient because it is associated with low transfection as well as sufficient permeability enhancement of membranes of the body cells. In this approach, the increasing uptake of the introduced DNA into the somatic tissues increases the APC influx within the areas of administration (van Drunen and Hannaman, 2010). The benefit associated with this process is that it triggers the immunity reactions such as the cytotoxic Tlymphocytes (CTL), which is an effective response. The method is also important when an analysis of the intracellular signals of DNA vaccines is required. Other methods under investigation for suitability include the needle-free systems, the gene gun and the mucosal introduction (Haynes et al., 2010; Rao et al., 2006; Torrieri-Dramard et al., 2011).

\section{Gene Expression and Production}

\section{Gene Expression}

Gene expression is a scientific process that entails the transformation of information from the particular gene for the purpose of production of essential products that are protein in nature. The mechanisms involved in gene expression engage the use of the genotypic characteristics to express the phenotype traits (Breaker, 2005). The non-protein-related products include the noncoded gene such as the functional RNA. However, it is worth noting that multicellular organisms, as well as the archaea and bacteria, can be used to generate the necessary structures and macromolecular forms of somatic organizations that are used in the process of gene expression. Gene delivery assists in the rectification and restoration of the mutated forms of the original structure of the gene. The process is achieved mainly through vaccination. Various methods can be used to express the genes that have been identified as exogenous. Viral vectors have the ability to incorporate the genetic payload into the genetic structure. Moreover, the viral vectors can also portray a gene in an episomal manner. It is essential to recognise that the vectors can offer useful viral-protein codes that are unfavourable for the somatic immune system to the extent of causing death. There also exists the possibility of the retroviral family members to be incorporated into the genome randomly resulting into self-mutation cases.

Another method of gene delivery is referred to as the naked DNA. This approach is associated with the genes with explicit expression. In most cases the process lasts for about 19 months. The technique is favourable because it can be employed within several tissues such as the muscles. Within the selected somatic tissues, the naked plasmid DNA plays a crucial role since the expected complications are minimised. The shortcomings associated with the use of the viral vectors such as mutation and possible death are eliminated when the plasmid DNA is used because the naked DNA allows the creation of equilibrium within the delivery paths (Bolhassani, 2011). The process of naked DNA is not only essential for gene delivery, but the technique is also employed when studying other physiologic processes. The method allows a straightforward approach to the problem through provision of evidence to create scientific conclusions concerning the conditions.

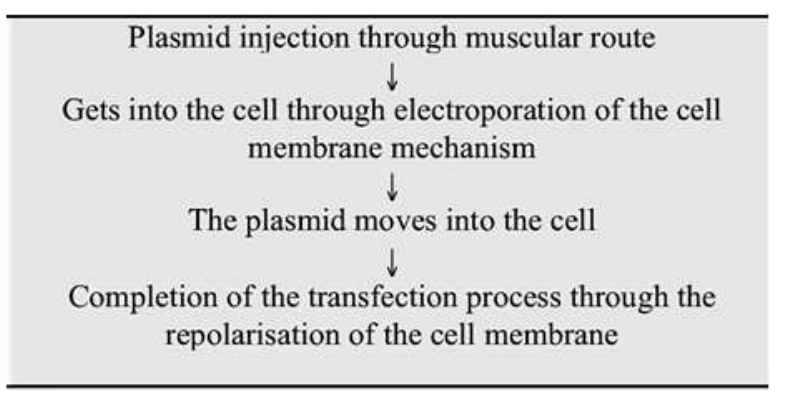

Fig. 1. A representation of the transfection process (Ahmad et al., 2012) 


\section{Gene Production of DNA}

The production of pcDNA3.1-eGFP involves the process of using the pcDNA3 (A1) as the backbone and the introduction of the pIRES2-eGFP (A2). The process is shown in the Sander Lab at University of California, San Diego (UCSD). The choice of the backbone is essential because it defines the success of the entire process. The inserted plasmid contains the eGFP that acts as the fluorescent reporter probe. The next stage is the analysis of the plasmids regarding the sequences.

In this technique, the chemically competent Escherichia coli (Invitrogen) enhances the pcDNA3 as well as the pIRES2-eGFP plasmids through transformation. During the pcDNA3.1-eGFP technique, the transformed cells grow whenever ampicillin is added to the backbone. A similar scenario is witnessed when kanamycin is added to the inserted plasmids. The Invitrogen is used to retrieve the DNA existing in the component cells employed in the technique. The developed chains are cut using the restriction enzymes and are then purified using the Invitrogen. The pcDNA3 backbone is also cut through electrophoresis gel. The pcDNA3 is also purified and the bands are then illuminated using the ultraviolet radiations to protect the sequences against possible mutation (Mezo et al., 2012).

PCR method is necessary because it helps in determining whether the bands have been inserted successfully. The primers applied in PCR method are similar to the clone inserted to the sequences that are used in the production of pcDNA3-eGFP plasmids in modern genetic engineering. The process of expressing the plasmid in mammals' cells involves the transfection of the pcDNA3-eGFP into 3T3 fibroblasts carried out using Mirus (TansIT transfection kit). The transfection process is summarised in Fig. 1. The fibroblasts are usually grown to about $80 \%$ confluence. Such cells are analysed for about 1-2 days after the transfection to ascertain the existence of eGFP using the fluorescence microscope.

\section{Production of the pDRIVE-Egfp}

The process of the production of pDRIVE-Egfp involves the pDRIVE-myoglobin and pDRIVE-desmin plasmids that are acquired from the Invitrogen to achieve the particular expressions. In this method, the pDNA is propagated through an overnight culturing period. This molecule undergoes cleaning, separation of the DNA backbone and the $L a c Z$ gene, which takes place through electrophoresis and also polymerase chain reaction (PCR) cloning and purification.

The pure band pDNA produced is then transformed into useful chemically competent cells. The pDNA produced is then mini-prepped and kept at a temperature of about $-20^{\circ} \mathrm{C}$ to ensure that the chances of internal transformation and mutations are eliminated through keeping pDNA inactive.
Moreover, there is always the need to affirm the presence of the inserted enhancements for purification and segregation. Therefore, two diagnostic tests are conducted. The process of gel electrophoresis is done to segregate the insertions from the backbone. Moreover, the course of ascertaining the added substances involves the examination of the position of the eGFP in the gel. This is to ensure that the eGFP has been inserted in the right place. PCR analysis using the process of forward Shable and reverse NcoI primers process is carried out to determine the orientation of the eGFP. The process is complicated and requires keen examination of each process and sequences generated regarding their respective position and the combinations of the insertions. However, it is a reliable technique for creating the pDRIVE-eGFP.

\section{Immune Response in Early Life}

Monocytes and macrophages, in the early life of organisms, have been linked to the limited expression of the major histocompatibility complex (MCH). The simulator molecules have been associated with the nature of the responses in embryogenesis and infants during development. Moreover, the decrease in production and action of the antigen proteins and transformed cytokine can be traced back to the inadequate action of the monocytes and macrophages. The antigen presenting cells' (APC) activity at birth and their development during the embryogenesis are slow and not advanced. The limited effect of the APCs contributes to the limitations associated with the induced immune responses within the somatic tissues in early life. It has been scientifically proven that the natural killer (NK) cells are significantly higher during birth, but their functionality is limited during the first afterbirth weeks. The complexity emanates from the need to understand the origin of the insufficiency of the intrinsic growth depicted in early life and embryogenesis. However, slow responses and transfection have been linked to the environment during the external administration of DNA vaccines. The nature of the responses depends on whether the host is an embryo, an infant, or an adult.

Moreover, the body systems of newborns and embryos can produce antibody reactions to respond to the pathogenic agents or the introduced vaccines. It is essential to note that IgG responses are slower in infants compared to adults when triggered in a reaction against infectious viruses. The early life and development have also been linked to a lower avidity of the vaccine antibody for specific immunity enhancement (Epstein et al., 2000). The slow rate of the maturity of the antibodies is due to the insufficient affinity of the T-cells among the infants and the developing embryo. The scenario explains why the immune system of children and expectant adults are supplemented by an external enhancement. Responses such as the reaction to carbohydrate factors similar to the bacterial immunity capsules and the viral glycosylated 
proteins are not sufficiently developed and pronounced in early life. However, the cases of the immune responses of the antibodies during early development are not related to the number of the B-cells in the somatic structures of infants or developing embryos. Nevertheless, the effects of DNA vaccination are notable with expectant adults due to the nature of influence associated with the process of embryogenesis.

\section{DNA Vaccination and Embryogenesis}

DNA vaccinations have been used to modify the viability of the organisms to their environment based on their genetic combination and structure. The process is carried out because of the need to transform the immune system to create sustainable responses within the somatic tissues and cells. However, the effect of such vaccinations produced through the mechanisms discussed above has a drastic effect on the process of embryo development as discussed below.

\section{DNA Vaccination and the Humoral Reactions}

DNA vaccines affect the kinetics of the antibody response within the embryo. The humoral reactions after the introduction of the vaccine last longer than the circumstances when the recumbent proteins enter the body. One humoral reaction example in cancer cells can be seen in Figure 2. The prolonged period occurs because the launch of DNA vaccines into the embryo triggers an extended response to the effects of viruses, such as hepatitis B. These vaccines end up creating a lifelong sustainable response that offers protection of the body against the influenza haemagglutinin. Such medical advancement has been ascertained in experiments conducted on mice and the effect of the vaccine has been succinctly studied (Ming-Derg et al., 2009). DNA vaccines transfer the cells responsible for antibody secretion from the embryo under development to the spleen and the bone marrow. The migration elongates the period of the antibody production and boosts the body's immunity. However, the rate at which the vaccines enhance protection is slower than that when the natural recombination does. Therefore, the time taken for the peak production of the antibodies to be attained after DNA vaccinations is longer than that during the natural production. The delayed attainment of the peak production is encouraged by the antigen levels in the body during administration (Nguyen et al., 2009).

DNA vaccination therapy supports the primary and secondary stages of the production of the antibodies. In expectant adults with hepatitis, the DNA vaccines containing the HBV protein give rise to the manufacture of interferon gamma cells that alter the development of the embryo. The gamma interferon produced is specific in nature. The T-cells against the inner envelope antigens are also presented in such a scenario.

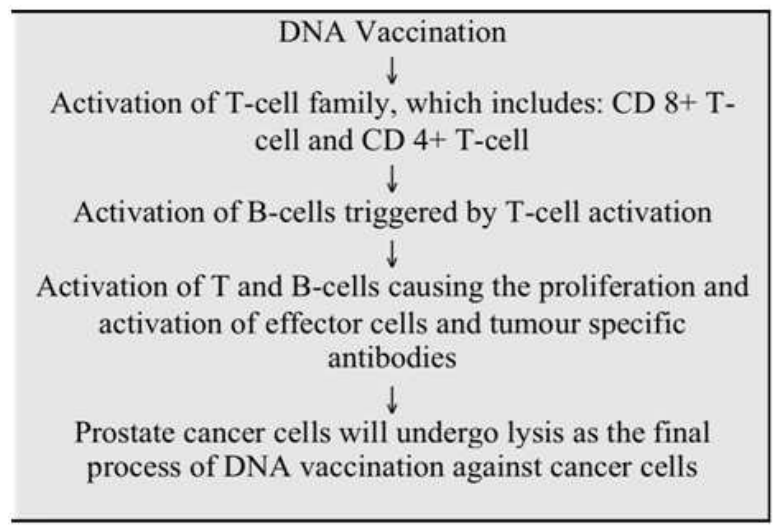

Fig. 2. Humoral reaction process against cancer cells after administration of DNA vaccines (Ahmad et al., 2012)

Worth noting is that the immunity of the expectant adults will not be in a position to counter the effects of the HBV injection (Bergman et al., 2003). Whenever pregnant adults are given DNA vaccines, the antibody production processes are altered. Several factors define the nature of the responses that are displayed by antibodies when DNA vaccines are introduced in pregnant individuals. The encoded antigens determine the factors and conditions associated with the responses of the antibodies. The nature of the response is also affected by the location of the gene that is being expressed. Other factors include the frequency of the introduction of DNA vaccines in the body, the dosages and the methods of administration. It is essential to understand that the location of the antigens being expressed can be intracellular or secreted (Rovero et al., 2000).

\section{DNA Vaccination and Inheritance Risks}

DNA vaccination is associated with the property of eliminating the inheritance risks during the embryo development among the expectant adults. The vaccines induce and express the responses of the CTLs during the development of the embryo without altering the genetic structure of the developing child. The fetus is protected from possible genetic recombination that may contain hereditary complications. However, the process permits the CTL epitopes that work against the actions of the immune-dominant and the immunerecessive epitopes. This, therefore, resembles a natural infection (Hooper et al., 2000). Thus, DNA vaccination among expectant adults can be used to control the deleterious effects of the epitopes. The control offers body immunity and, thereby, safeguarding the growth and development of the embryo. The cells can recognise the minute and simple peptides and molecules that are complex in nature within the somatic system of the developing fetus. The peptides are the 
result of the endogenous cytosolic proteins, which are degraded and delivered to endoplasmic reticulum primary module of the MHC class. Thus, the DNA vaccines allow the body of the expectant adults to react to the intended modifications without affecting the growing fetus regarding the genetic combination. This effect occurs because the gene production enhances the CTL responses (Ming-Derg et al., 2009).

\section{Advantages and Disadvantages of DNA Vaccination}

There are notable advantages associated with the application of DNA vaccines among living organisms as summarised in Table 1. Based on the conducted experiments and investigations, the introduction of the viable DNA modification in the somatic genetic structure of the hosts creates a more favourable immune response system when compared with the ordinary immune boosters. The vaccines have limited subunit risks for secondary infections (Robinson and Pertmer, 2000). The traditional immune enhancement processes are faster but not reliable for sustainable pathogenic responses in future infections. The vaccines allow the expression of the antigen through the presentation of MHC class I and II. DNA vaccination has the potential of polarizing the T-cells for efficient MHC classes' viability. The T-cells plays a vital role in triggering the specific responses during the antibody reactions in early life and within a developing embryo. The vaccines are more stable and adaptive as well as cheap to produce. Pathogenic viruses have been known to occasionally undergo mutation processes; however, DNA vaccines can be easily transformed to fit the intended pathogenic structural alterations.

Besides, the traditional vaccines cannot offer the needs responsible for peptide synthesis. Such avenues and mechanisms for the expression of the recombinant proteins are provided whenever the DNA vaccines are used. The vaccines offer the purification of the proteins as well as the use of sufficient adjuvants within the structures (Sedegah et al., 1994). The immunogenicity of DNA vaccines is persistent and lasts for a longer period compared to the traditional vaccination methods of which the sustainability is limited. The application of in vivo expressions guarantees the resemblance of the proteins to the eukaryotic structures but offers room for significant modification of the strand features. This, therefore, increases the effectiveness of the responses against pathogenic viruses.

Nevertheless, DNA vaccines have notable shortcomings. The vaccines are protein-based and there exists unreliable applications whenever the antigens are not protein-based. A good example involves the scenario characterised by bacterial polysaccharides. DNA vaccines cannot be applicable in such immunity evading characteristics. There are possibilities of the genes affecting the growth of the cells. Only one DNA vaccination has been approved to be used in humans because of the fear that the genetic modification could lead to abnormal growth within the targeted somatic cells. Moreover, the vaccines can induce a tragic response against the DNA structure of the hosts resulting in the possible autoimmune destruction of the normal DNA sequence. Experiments have also shown that DNA vaccines are associated with the production of the proteins that are parasitic and bacterial in nature that is not favourable for the healthy body's physiological processes (Robinson and Pertmer, 2000).

\section{Enhancement of the DNA Vaccination Responses}

The effectiveness of DNA vaccines can be enhanced when the developed DNA structures can be stabilised to protect them from the effects of degradation.

Table 1. Summary of advantages and disadvantages of DNA vaccines

\begin{tabular}{ll}
\hline Advantages & Disadvantages \\
\hline $\begin{array}{l}\text { Creates a more favourable immune response system } \\
\text { (Robinson and Pertmer, 2000) }\end{array}$ & $\begin{array}{l}\text { Unreliable applications whenever the antigens are not protein-based } \\
\text { (Robinson and Pertmer, 2000) }\end{array}$ \\
$\begin{array}{l}\text { Limited subunit risks for secondary infections } \\
\text { (Robinson and Pertmer, 2000) }\end{array}$ & $\begin{array}{l}\text { Fear that the genetic modification could lead to abnormal growth } \\
\text { within the targeted somatic cells (Robinson and Pertmer, 2000) }\end{array}$ \\
$\begin{array}{l}\text { Reliable for sustainable pathogenic responses in future } \\
\text { Can induce a tragic autoimmune response against the DNA structure of } \\
\text { infections (Robinson and Pertmer, 2000) }\end{array}$ & $\begin{array}{l}\text { Associated with the production of the proteins that are parasitic and } \\
\text { bfficient MHC classes' viability due to polarization } \\
\text { (Sedegah et al., 1994). }\end{array}$ \\
$\begin{array}{l}\text { More stable and adaptive (Sedegah } \text { et al., 1994). } \\
\text { Cheap (Sedegah et al., 1994). }\end{array}$ & \\
$\begin{array}{l}\text { Easily transformed to fit the intended pathogenic } \\
\text { structural alterations (Sedegah } \text { et al., 1994). }\end{array}$ & \\
$\begin{array}{l}\text { Immunogenicity of DNA vaccines is persistent and } \\
\text { lasts for a longer period (Robinson and Pertmer, 2000) }\end{array}$ & \\
\hline
\end{tabular}


The process of delivery should be strengthened to increase the accuracy of the manner in which the DNA is introduced into the cells that present the antigens (Robinson and Pertmer, 2000). Scientific analysis has shown that the coating of the DNA with the biodegradable positively charged ion particles such as the polylactide-co-glycolide molecules, which are generated by the cetyltrimethylammonium bromide is effective in this function. The decision of coating the DNA is an efficient process that ensures the CTL is portrayed as the recombinant virus for the vaccine within the tissues of the body. Moreover, DNA vaccines have been improved by using the alphavirus recombinants vectors. The process involves the insertion of the gene responsible for the encoding of the targeted antigen into the replicon alphavirus. The introduction of the gene into the DNA structure does not alter the nonstructural gene replicate but replaces the structural ones (Robinson and Pertmer, 2000).

\section{Conclusion}

The use of DNA vaccines awakens the somatic cells of the host to produce the antigens that will assist the body to prepare to fight infections through preventive measures triggered by the vaccine. Through DNA vaccination, dominant infections of animals have been controlled and the hope to eradicate the human infections and diseases through the same technique is high. In humans, the aspect of DNA vaccination is not entirely advanced and experimental studies are being conducted to ascertain the possibility of existing solutions to eliminate common bacterial, viral and parasitic infections as it has been shown with animals. In embryogenesis and early life, the monocytes and macrophages of organisms have been linked to the limited expression of the major histocompatibility complex $(\mathrm{MCH})$. The simulator molecules have been associated with the nature of the responses during development. DNA vaccines affect the kinetics of the antibody response within the embryo. The humoral reactions after the introduction of the vaccines last longer than the circumstances when the recumbent proteins enter the body. Besides, DNA vaccination supports the primary and secondary stages of the production of the antibodies. Moreover, DNA vaccination is associated with the property of eliminating the inheritance risks during embryo development among the expectant adults. The vaccines express the responses of the cytotoxic T-cells by inducing the cytotoxic Tlymphocytes during the development of the embryo without altering the genetic structure of the developing child.

\section{Acknowledgment}

Special thanks to Dr. Budiman Bella and Dr. Fera Ibrahim for supporting the preliminary study in IHVCB Laboratory. Special thanks to the Dean of the Faculty of Medicine, Universitas Indonesia, for giving the permission letter to do the research.

\section{Funding Information}

The research was funded and supported by "Hibah Awal DRPM UI Year 2013."

\section{Author's Contributions}

This paper was written and developed based on the ideas from Ria Margiana.

\section{Ethics}

There are no ethical issues that may arise after the publication of this manuscript.

\section{References}

Ahmad, S., P. Sweeney, G.C. Sullivan and M. Tangney, 2012. DNA vaccination for prostate cancer, from preclinical to clinical trials where-we stand? Genetic Vaccines Therapy, 10: 1-9.

DOI: $10.1186 / 1479-0556-10-9$

Alam, S. and D.G. McNeel, 2010. DNA vaccines for the treatment of prostate cancer. Expert Rev. Vaccines, 9: 731-745. PMID: 20624047

Barouch, D.H., S. Santra, J.E. Schmitz, M.J. Kuroda and T.M. Fu et al., 2000. Control of viremia and prevention of clinical AIDS in rhesus monkeys by cytokine-augmented DNA vaccination. Science, 290: 486-492. PMID: 11039923

Bergman, P.J., J. McKnight, A. Novosad, S. Charney and J. Farrelly et al., 2003. Long-term survival of dogs with advanced malignant melanoma after DNA vaccination with xenogeneic human tyrosinase: A phase I trial. Clin. Cancer Res., 9: 1284-1290. PMID: 12684396

Bolhassani, A., 2011. Improvement of different vaccine delivery systems for cancer therapy. Molecular Cancer, 10: 2-20. PMID: 21211062

Breaker, R.R., 2005. Gene expression control: Harnessing RNA switches. Gene Therapy, 12: 725-726. DOI: $10.1038 /$ sj.gt.3302461

Desmet, C.J. and K.J. Ishii, 2012. Nucleic acid sensing at the interface between innate and adaptive immunity in vaccination. Nat. Rev., 12: 479-491. PMID: 22728526 
Epstein, S., S. Fuchs, Y. Zhou, R. Baffour and R. Kornowski, 2000. Therapeutic interventions for enhancing collateral development by administration of growth factors: Basic principles, early results and potential hazards. Cardio. Res., 49: 532-542. PMID: 11166266

Faurez, F., D. Dory, V. le Moigne, R. Gravier and A. Jestin, 2010. Biosafety of DNA vaccines: New generation of DNA vectors and current knowledge on the fate of plasmids after injection. Vaccine, 28: 3888-3895. PMID: 20371391

Haynes, J.R., D.E. McCabe, W.F. Swain, G. Widera and J.T. Fuller, 1996. Particle-mediated nucleic acid immunization. J. Biotechnol., 44: 37-42. PMID: 8717384

Hooper, J.W., D.M. Custer, C.S. Schmaljohn and A.L. Schmaljohn, 2000. DNA vaccination with vaccinia virus $L 1 R$ and A33R genes protects mice against a lethal poxvirus challenge. Virology, 266: 329-339. PMID: 10639319

Ingolotti, M., O. Kawalekar, D.J. Shedlock, K. Muthumani and D.B. Weiner, 2010. DNA vaccines for targeting bacterial infections. Expert Rev. Vaccines, 9: 747-763. PMID: 20624048

Mezo, A.R., K.A. McDonnell, S.C. Low, J. Song and T.J. Reidy et al., 2012. Atrial natriuretic peptide-Fc, ANP-Fc, fusion proteins: Semisynthesis, in vitro activity and pharmacokinetics in rats. Bioconjug Chem., 23: 518-526. PMID: 22263969

Ming-Derg, L., Y. Meng-Chi, L. Chiu-Mei, T. Cheng-Fen and W. Chun-Chin et al., 2009. Genetic vaccines and therapy. Gov. J.

Nguyen, D.N., J.J. Green, J.M. Chan, R. Langer and D.G. Anderson, 2009. Polymeric materials for gene delivery and DNA vaccination. Adv. Mater., 21: 847-867. DOI: 10.1002/adma.200801478

Rao, S.S., P. Gomez, J.R. Mascola, V. Dang and G.R. Krivulka et al., 2006. Comparative evaluation of three different intramuscular delivery methods for DNA immunization in a nonhuman primate animal model. Vaccine, 24: 367-373. PMID: 16194587

Redding, L. and D.B. Weiner, 2009. DNA vaccines in veterinary use. Expert Rev. Vaccines, 8: 1251-1276. PMID: 19722897

Rekvig, O.P. and J.C. Nossent, 2003. Anti-doublestranded DNA antibodies, nucleosomes and systemic lupus erythematosus: A time for new paradigms? Arthritis Rheum., 48: 300-312. PMID: 12571837
Robinson, H.L. and T.M. Pertmer, 2000. DNA vaccines for viral infections: basic studies and applications. Adv. Virus Res., 55: 1-74. PMID: 11050940

Rovero, S., A. Amici, E. Di Carlo, R. Bei and P. Nanni et al., 2000. DNA vaccination against rat her-2/Neu p185 more effectively inhibits carcinogenesis than transplantable carcinomas in transgenic $\mathrm{BALB} / \mathrm{c}$ mice. J. Immunol., 165: 5133-5142.

PMID: 11046045

Saade, F. and N. Petrovsky, 2012. Technologies for enhanced efficacy of DNA vaccines. Expert Rev. Vaccines, 11: 189-209. PMID: 22309668

Sedegah, M., R. Hedstrom, P. Hobart and S.L. Hoffman, 1994. Protection against malaria by immunization with plasmid DNA encoding circumsporozoite protein. Proc. National Acad. Sci. USA, 91: 9866-9870. PMID: 7937907

Shimamura, M., N. Sato and R. Morishita, 2011. Experimental and clinical application of plasmid DNA in the field of central nervous diseases. Curr. Gene Ther., 11: 491-500. PMID: 22023479

Silva, C.L., V.L. Bonato, R.R. dos Santos-Junior, C.R. Zarate-Blades and A. Sartori, 2009. Recent advances in DNA vaccines for autoimmune diseases. Expert Rev. Vaccines, 8: 239-252. PMID: 19196203

Spiegelberg, H.L., K. Takabayashi, L. Beck and E. Raz, 2002. DNA-based vaccines for allergic disease. Expert Rev. Vaccines, 1: 169-177. PMID: 12901556

Torrieri-Dramard, L., B. Lambrecht, H.L. Ferreira, T. van den Berg and D. Klatzmann et al., 2011. Intranasal DNA vaccination induces potent mucosal and systemic immune responses and crossprotective immunity against influenza viruses. Mol. Ther., 19: 602-611. PMID: 20959813

Ulmer, J.B., J.J. Donnelly, S.E. Parker, G.H. Rhodes and P.L. Felgner et al., 1993. Heterologous protection against influenza by injection of DNA encoding a viral protein. Science, 259: 1745-1749. PMID: 8456302

Van Drunen, H.S. and D. Hannaman, 2010. Electroporation for DNA immunization: Clinical application. Expert Rev. Vaccines, 9: 503-517. PMID: 20450325 